\title{
Microeconometric Analysis of the Determinants of Savings Bahaviour in Zimbabwe: 2009-2012
}

\author{
Chikoko Laurine ${ }^{1}$, Pierre Le Roux ${ }^{2} \&$ Dzingirai Canicio ${ }^{3}$ \\ ${ }^{1}$ Department of Banking and Finance, Midlands State University, Gweru, Zimbabwe \\ ${ }^{2}$ Department of Economics, Nelson Mandela Metropolitan University, South Africa \\ ${ }^{3}$ Department of Economics, Midlands State University, Gweru, Zimbabwe \\ Correspondence: Chikoko Laurine, Department of Banking and Finance, Midlands State University, Gweru, \\ Zimbabwe. Tel: 263-773-095-507. E-mail: chikokol@msu.ac.zw
}

Received: January 9, 2013 Accepted: March 6, 2013 Online Published: April 27, 2013

doi:10.5539/ijbm.v8n10p159 URL: http://dx.doi.org/10.5539/ijbm.v8n10p159

\begin{abstract}
A high savings culture is a foundation of a sustainable national social, sound financial and economic policy. The main objective of this paper was to gain insights on savings behaviour by individuals in Zimbabwe after the adoption of the multiple currency exchange rate system. A micro econometric approach which took into account of individual heterogeneity was adopted. The selected individual characteristics inputted in the logit model as savings predictors were age, marital status, religion, education, position in the household, household size, type of accommodation, place of accommodation, employment status, income, number of people employed in the household, and expenditure per month. The goal was to understand which variables authorities and commercial banks might need to target in order to promote a savings culture in Zimbabwe after most people lost their savings in the banks due to hyperinflation. Secondly, we investigated within a sample of savers what determined the number of times individuals would deposit money in the bank by use of Poisson regression model. Finally, we came up with a savings predicting model which helps banks identify targeted customers based on the individual characteristics. The results revealed that majority of the variables were significant as predictors to savings. However, income level proved to be the most significant variable as it had the least probability value. To promote a saving culture, banks in Zimbabwe may need to consider bringing in differentiated and tailor made products for the high net worth and the mass market.
\end{abstract}

Keywords: Zimbabwe, microeconometric, savings behaviour, commercial bank, logit, poisson

\section{Introduction}

The government of Zimbabwe deliberately allowed the use of a multiple currency exchange rate system (MPC), which was adopted on 30 January 2009. The multiple currency system allows trade to be completed using major trading currencies, for example, the United States Dollar, Pound Sterling, South African Rand, and the Botswana Pula. The new regime helped restore price stability and restart financial intermediation (MOF 2010, RBZ 2010). Nevertheless, it took a lot of effort to attract deposits back into the system in Zimbabwe due to loss aversion phobia after most people lost their savings in the banks due to hyperinflation. Given this background, it is important to understand how a savings culture can be promoted in Zimbabwe. To achieve that, it is important to understand fundamentals that determine savings.

Aggregate national saving is composed of individual, corporate and public savings. Compared with developed world, Zimbabwe has no official household databank for each individual household except time series data on national aggregate saving, mostly analysed using macro-econometrics models premised on homogeneity assumption. Homogeneity assumption induces error of measurement bias which renders the parameters inconsistent and asymptotically inefficient which might violate the Gauss-Markov assumptions. Post 2008 era, household saving was an increasingly crucial issue for families, individuals and industry resuscitation in Zimbabwe, given that almost everything was lost during the pre 2008 era due to hyperinflation (RBZ, 2009; Ministry of Finance, 2009).

Over the period 2009-2011 individual deposit rate averaging 1\% was far below the average economic growth rate of $4 \%$ (RBZ, 2012). To make matters worse, in 2010, the household savings with financial institutions 
declined sharply by $10 \%$ (ibid), justifying Beverly et al.'s (2003) assertion that during economic downturn and liquidity crisis periods, individual savings and asset accumulation are universally viewed as desirable goals for economic turnaround stimuli. Recent shifts in the structure of developing countries economies in general and Zimbabwe in particular have brought debate on the possible solutions to such economic quagmires and, significance and determinants of household saving to the center of the policy arena (Attanasio and Bank, 2001; Fisher and Montalto, 2011). This has stimulated a debate on the importance and adequacy of household savings for social and economic growth financing through investment an injection in the circular flow of income. Up to now, no consensus exist among economists and policy makers on the microeconomic determinants of observed household saving behavior and possible drivers of difference in deposit times within a month.

A large body of literature studies savings from a macroeconomic perspective. Unfortunately, macroeconomic approaches ignore the heterogeneity in consumers which realistically reflect different influences of savings behaviour by consumers. As a result, a micro-econometric approach is highly recommended inorder to have insights on savings behaviour by individuals in Zimbabwe after the adoption of the MPC. A micro econometric approach which took into account the individuals' heterogeneity was adopted. The objective was to understand which variables authorities and commercial banks would need to target in order to promote a savings culture. Secondly, we investigated within a sample of savers what determined the number of times that an individual deposit money in the bank by use of Poisson regression model. Lastly, our objective was to come up with a savings predicting model to be able to identify targeted customers based on the individual characteristics.

\section{Literature Review}

Savings are defined as a leakage out of the circular flow of income meaning it is that part of income that has been left unconsumed and thus not given back to the circular flow of income but is rather spared for future use (Mankiw 2007). The forgoing of present consumption for a higher level of future consumption is a better way of defining savings. Savings also are done through the depositing of money in banks or other investment where a future greater return is expected thus savings should equal investment without this the purpose of savings is distorted.

Revealing the micro determinants of the aggregate saving rate is a paramount prerequisite in designing a number of crucial policy interventions, from a tax and social scrutiny system to the layout of financial markets regulation. Therefore it's not surprising that the analysis of saving behaviour has become one of the central issues in empirical micro and macro-economics (Deaton, 2005). Savings are an inherent part to all players of the economy. Individuals seek to smooth out consumption over time, saving in "good times" to consume in "bad times". The precautionary motive for savings fundamentally affects the saving behaviour. Individuals benefit from saving mostly for precautionary reasons, the fact that the future is uncertain means that action should be taken before hand to hedge against any future adversities death. The corporate world realises its potential to boost the economy activities by accumulating capital stocks through the conversion of resources thus in doing so financial institutions and other firms hedge themselves against uncertainties like during a time of economic downfall, savings can also be used for the paying out of dividends and thus increasing the welfare of shareholders. The government also needs savings so as to finance the government's public sector borrowing requirement (i.e. the funds in excess of taxation that the government requires to fund its activities). It is from national savings that the government is able to improve the welfare of its citizens e.g. the granting of pension funds, drought relief incentives and etc. Researches recognise saving as a crucial factor for the economic development as it enables conversion of resources into capital. Strong saving performance is crucial for macroeconomic balance.

Two of the most well-known theories of savings are the Life Cycle Hypothesis and the Permanent Income Hypothesis.

\subsection{The Life Cycle Hypothesis}

Life Cycle theory appears first in a paper by Modigliani and Brumberg (1954). They worked out a theory of spending based on the idea that people make intelligent choices about how much they want to spend at each age, constrained only by the resources available over their life time. For each individual, it is assumed that an increase in life-time resources leads to a proportionate increase in consumption in all periods of life. As a result, consumption is proportional to lifetime resources or, what is more or less the same thing, to average income over the life time (Deaton, 2005). One of the main implications of the Life Cycle model is that individuals can separate consumption from income, that is, consumption is not affected by the timing of income. Individuals tend to dissave when they are young, because of relatively low levels of income and high expenditures due to household formation while they tend to save in their middle years, with asset holdings reaching their maximum at retirement age, an indication of non-linear relationship between saving and age. Individuals again dissave 
during retirement by using their accumulated assets, which are entirely exhausted at death. This justify the inclusion of age and age-squared in the model.

The Life Cycle theory leads to important and more advanced predictions about the economy as a whole, for example that national saving depends on the rate of growth of national income, not its level, and that the level of wealth in the economy bears a simple relation to the length of the retirement span. Further, it is the Life Cycle Hypothesis that helps us think about a number of important policy questions about which we would otherwise have very little to say, for example how societies should collectively make provision for the increasing numbers of elderly and how does government provision interact with private provision (Deaton, 2005). While there have been many challenges to the theory of consumption over the years, the Life Cycle Hypothesis remains an essential part of economists' toolbox because it helps us to think about things such as the private and public provision of social security, the effects of demographic change on national saving and the role of saving in economic growth. It is sometimes questioned whether the Life Cycle Hypothesis of savings is still supported empirically. In many aspects it has been rejected.

\subsection{The Permanent Income Hypothesis}

Dynan et al. (2004) asserts that, an analyses of standard economic approach to saving implies that people with high temporary income will save more to compensate for lower future income, with those with temporarily lower income tend to save less in anticipation of higher future income. Even though saving rates are highly volatile with lifetime income, individuals with high current income, ceteris paribus, are expected to be thriftier than those with low current income (Friedman, 1957).

The Permanent Income Hypothesis follows the logic of the Life Cycle Hypothesis; that consumption should not depend on current income alone. Unlike the Life Cycle Hypothesis, which anticipates that income follows a regular pattern over a lifetime, the Permanent Income Hypothesis instead emphasizes that people experience random and temporary fluctuations in income from year to year. Friedman divides income into a permanent part, which is the income people expect to persist into the future, and transitory part, which is the income that people do not expect to persist. More simply, permanent income is average income and transitory income is deviations from this level of income. Friedman argued that consumption should primarily depend on permanent income because people save and borrow to smooth consumption in response to transitory changes in income. If shocks to income are permanent, then all future levels of income will be revised upwards or downwards by the same amount leading to a change in consumption, which is equal to the change in current income. Central is that the consumption plan does not depend on the transitory components. Friedman argued that individuals with high permanent income consume the same fraction of permanent income as individuals with low permanent income.

DeJuan et al. (2006) and also Dynan et al. (2000) took up several studies of the Permanent Income Hypothesis and conclude that the evidence for the hypothesis is mixed at best, with some studies supporting it and some studies rejecting it. A problem with the theorem, emphasized by Meghir (2004), is that the loose definition of permanent income makes it difficult to measure. However, the reason that the Permanent Income Hypothesis has endured so much is that, beyond its simple intuitive appeal, it focuses on inter-temporal optimization of consumer behavior, which is logical and consistent.

The determinants of savings generally and the specific effects of government policies on savings is pivotal force in investment and economic growth. The level of savings depends on the marginal propensity to save of the players of the economy; this is the proportion of a small change in disposable income that would be saved, instead of being spent on consumption. It is computed by dividing the change in savings by the change in disposable income that caused the change. Savings also depend on the willingness and ability of individuals and corporate to save.

Few studies investigated individuals' savings using micro econometric approach due to lack of data. According to our knowledge, household saving behaviour in Zimbabwe at micro level has been carried out. From the few studies that have analysed household saving behaviour at micro level, some of the included variables are as follows.

\subsection{Income Growth and Income Level}

Income is also a major determinant of the level of savings. Subsistence-consumption theories suggest that countries with higher income levels tend to have a higher saving rate; the aggregate savings will increase in response to an increase in income growth and vice-versa when income is low consumption tends to be high.

\subsection{Demographic Variables}

Age and geographical distribution tend to have an impact on savings. Consumers are considered to have negative 
savings when young and with low income, positive savings during their productive years and negative savings when they are old and retired (Modigliani, 1970). Another demographic variable is the urbanization ratio, defined as the percentage of the total population living in urban areas. This variable is also expected to have a negative impact on saving

\subsection{Employment}

This is an important determinant of savings since the more the people employed the more their ability to generate income that would be saved if not fully consumed, coming from another angle, if there are more people employed at one household this means that the income of one person is not exhausted in consumption but can be partly consumed and the remainder is postponed

It is clear that various influences are suggested but unfortunately these provide ambiguous results. Our econometric specification allows us to test responses of selected individual characteristics which were inputted in the logit model as savings predictors were age, marital status, religion, education, position in the household, household size, type of accommodation, place of accommodation, employment status, income, number of people employed in the household, and expenditure per month. In addition, we investigated within a sample of savers what determined the number of times the individual deposit money in the bank by use of Poisson regression model.

\section{Data and Methodology}

\subsection{Empirical Model}

Saving can be defined as the change in wealth over a certain time period or as the difference between income and consumption (Beverly et al., 2003). It is a complement to consumption, if income and wealth are held constant (Xiao and Olson, 1993). In this research, we used a logit model and a Poisson regression model. For the logit model we generated the saving variable by first subtracting amount consumed from the household head's disposable income. Income in excess of consumption was used in the current study to create a dichotomous depended variable; SAVED, coded as 1 if the amount saved is at least US\$100 and 0 otherwise. The logit model is used to come up with the savings predicting model. The logistic function is as follows

$$
f(S)=\frac{1}{1+e^{-S}}-\infty \leq S \leq \infty
$$

Clearly, $0 \leq \mathrm{f}(\mathrm{S}) \leq 1$

The variable $S$ represents the savings determining factors while $f(S)$ represents the probability of an event occurring. The variable $\mathrm{S}$ is a measure of the total contribution of all the savings determining factors used in the model and is known as the logit. The above function can be transformed into the following:

$$
S=\beta_{0}+\beta_{i} X_{i}+\varepsilon_{i}
$$

Where: $S=$ savings; $\beta_{0}=$ automous savings; $\beta_{i}=$ vector of regressors parameters; $X_{i}=$ a vector of socio-economic and demographic characteristics and $\varepsilon_{i}=$ error term.

Using the logit regression model given above, the likelihood of saving is modeled as a function of the household's income and several other covariates prescribed by literature to affect one's saving behaviour.

The Poisson regression model is a count model which we used to regress the number of times one makes deposits. The model in its complete version is as follows:

$$
N S=\beta_{0}+\beta_{i} X_{i}+\varepsilon_{i}
$$

NS is number of times one makes a deposit within a month. Other parameters are as defined earlier on.

\subsection{Poisson Estimation Methodology}

The response variable NS, the number of times one makes a deposit within a month, is a non-negative integer or count which is explained by a set of covariates (Xs) being explanatory variables from equation 2. According Cameron and Trivedi (2005), Poisson is a point process for the occurrence of the event of interest. This implied the Poisson distribution modeled the number of times one makes deposit (NS), with density 


$$
\operatorname{Pr}(N S=n s)=\frac{e^{-\mu} \mu^{n s}}{n s !} ; \mathrm{ns}=1,2,3
$$

Where $\mu$ is the intensity or rate parameter. The distribution is refered as $P(\mu)$. Based on Green $(2005: 121)$, the two moments which show equidispersion are

$$
E(Y)=\mu \text { and } \operatorname{Var}(Y)=\mu
$$

Poisson regression is one of the robust models for the analysis of discrete data that are based on the assumption that the dependent variable $N S$ is distributed as Poisson, and its logarithm is a linear function of the independent variables (Green 2008a), which by parameterising the relation between the mean parameter $\mu$ and covariates gives:

$$
\mu_{i}=e^{\beta_{o}+\sum_{j=i}^{k} \beta_{j} x_{j}} \mathrm{i}=1,2, \ldots, \mathrm{n}
$$

Where $\mu$ is the expected number of times individual household $i$ save.

$\beta_{0}$ is the intercept; $\beta_{j}{ }^{\prime} s$ and $X_{j}{ }^{\prime} s$ are vectors of regression coefficients and explanatory variables from equation (2) of model specification respectively and $k$ is the number of linear independent covariates in this case equal to 13. Since $\operatorname{Var}\left(y_{i} / x\right)=e^{\beta_{o}+\sum_{j=i}^{k} \beta_{j} x_{j}}$, by 4 and 5 , the Poisson regression model is heteroskedastic, which needs maximum likelihood estimation (MLE) to correct, whose log-likelihood function according to Cameron and Trivedi, 2005: 667, is as follows:

$$
\ln L\left(\beta_{j}\right)=\sum\left\{y_{i} \beta_{i} X_{i}-\exp \left(\beta_{i} X_{i}-\ln y_{i} !\right\}\right.
$$

The Poisson MLE, denoted by $\hat{\beta}_{p}$ is the solution to $\mathrm{k}$ non-linear equations (Green 2008a), corresponding to the first-order condition for maximum likelihood,

$$
\sum_{i=1}^{n}\left(y_{i}-\exp \left(\beta_{i} X_{i}\right)\right) X_{i}=0
$$

Since $X_{i}$ coefficiets $(\beta s)$ includes a constant term $\left(\beta_{0}\right)$ the residual $y_{i}-\exp \left(\beta_{i} X_{i}\right)$ sum to zero by (7). The log-likelihood function is globally concave; hence solving these equations Gauss-Newton or Newton-Raphson iterative algorithm yield unique parameter estimates, which are both consistent and asymptotically efficient. By standard maximum likelihood of correctly specified models, Cameron and Trivedi (2005) asserts that the estimator $\hat{\beta}_{p}$ is consistent for $\beta$ of each parameter and asymptotically normal with the sample covariance matrix

$$
\operatorname{Var}\left(\hat{\beta}_{p}\right)=\left(\sum_{i=1}^{n} \mu_{i} X_{i} X_{i}^{\prime}\right)^{-1}
$$

In the case where $\mu_{i}$ is of the exponential form (5). The frequency of saving by each household is a discrete variable, and the distribution saving between different households demonstrates asymmetrical distribution with a modal value of 0 , supporting the appropriateness of modeling saving frequency as a Poisson process. On the interpretation of Poisson regression coefficients, suppose $\beta_{i}$ is the coefficient of a 0-1 dummy variable like 
employment status or gender, then the exponentiation of this coefficient (i.e. $\exp \left(\beta_{i}\right)$ represents the proportionate

change in the frequency of saving when the value of the variable changes from 0 to 1 , ceteris paribus. Similarly, when $\beta_{i}$ is a Poisson regression coefficient for a continuous variable, like households' age, then $\exp \left(\beta_{i}\right)$ represents the proportionate change in the mean number saving times when the value of the predictor variable increases by one unit, holding everything else constant (Wang and Famoye, 1997).

The above interpretations are based on the variable coefficient sign and magnitude, but they can be interpreted in terms of incidence ratios (Greene 2005). Before we interpret the coefficients in terms of incidence rate ratios, we must address how we can go from interpreting the Poisson regression coefficients as a difference between the logs of expected counts to incidence rate ratios (IRR). In the interpretation above, Poisson regression coefficients were interpreted as the difference between the log of expected counts, where formally, this can be written as $\beta_{i}=\log \left(\mu_{X i+1}\right)-\log \left(\mu_{X i}\right)$, where $\beta$ is the regression coefficient, $\mu$ is the expected count of children ever born and the subscripts represent where the predictor variable (regressors), say $X_{i}$, is evaluated at $X$ and $X+1$ (implying a one unit change in the predictor variable X). Recall that the difference of two logs is equal to the log of their quotient, $\beta_{i}=\log \left(\mu_{X i+1}\right)-\log \left(\mu_{X i}\right)=\log \left(\frac{\mu_{X i+1}}{\mu_{X i}}\right)$ and therefore, we also interpreted the parameter estimate as the log of the ratio of expected counts: This explains the "ratio" in incidence rate ratios. In addition, what we referred to as a count can also be called a rate. By definition a rate is the number of events per time (or space), which our response variable, saving frequency qualifies. Hence, we also interpreted the Poisson regression coefficients as the log of the rate ratio: This explains the "rate" in incidence rate ratio.

Finally, the rate at which events occur is called the incidence rate; thus we arrive at being able to interpret the coefficients in terms of incidence rate ratios from our interpretation above. IRR enable us to compare and quantify the impact that an explanatory variable, taking into consideration its composition, have on the dependent variable. For example if a variable is binary like gender, it tells us how many times male household heads saves as compared to their female counterparts, ceteris paribus. According to Cameron and Trivedi (2005), for any model with exponential condition mean, of a linear model $E(y / x)=\beta X$, the coefficients $\boldsymbol{\beta} s$ are readily interpreted as the effect of a one-unit change in regressors on the conditional mean. Its differentiation yields:

$$
\frac{\partial E(y / x)}{\partial x_{j}}=\beta_{j} \exp (\beta X)
$$

Where the scalar $x_{j}$ denotes the $\mathrm{j}^{\text {th }}$ regressor, $\beta_{j}$ measures the relative change in $E(y / x)$ induced by a unit change in $x_{j}$, if $x_{j}$ is measured on log-scale, $\beta_{j}$ is elasticity. For the purpose of reporting a single response value, a good candidate is an estimate of the average response, $\frac{1}{n} \sum \frac{\partial E\left(y_{i} / x_{i}\right)}{\partial x_{i j}}=\hat{\beta}_{j} \times \frac{1}{n} \sum \exp \left(\hat{\beta} X_{i}\right)$ since the Poisson Regression Model has an intercept included it simplifies to $\hat{\beta}_{j} \bar{y}$. From 9, if $\beta_{j}$ is twice as large as $\beta_{k}$, then the effect of changing the $\mathrm{j}^{\text {th }}$ regressor by one unit is twice that of changing the $\mathrm{k}^{\text {th }}$ regressor by one unit.

\subsection{Diagnostic Tests}

The researcher only resort on one diagnostic test of over-dispersion as propounded by Cameron and Trivedi (2005). It determines whether the mean and variance are same, a case known as equi-dispersion. If that is the case, a Poisson Regression Model becomes the most appropriate estimation methodology and any form of deviation from this condition; a Negative Binomial Model becomes the most suitable estimation methodology. The test goes hand in hand with maximum likelihood and its P-value and sometimes pseudo R-squared. 


\section{Data Analysis and Results}

Table 1. Descriptive statistics

\begin{tabular}{lcccccc}
\hline Variable & Observation & Mean & St Dev & Min & Median & Max \\
\hline Age & 350 & 38.98 & 13.1890 & 18 & 37 & 81 \\
Income & 350 & 955.4376 & 1084.977 & 30 & 530 & 7000 \\
Expenditure & 350 & 763.9105 & 842.6512 & 30 & 450 & 6000 \\
Savings & 350 & 191.5271 & 500.8137 & -2000 & 100 & 3000 \\
\hline
\end{tabular}

On average the respondents were 39 years old, with the average income being $\$ 955.00$ with the majority having income of $\$ 530.00$. The results also revealed that in Zimbabwe the amount of expenditure was around $\$ 760.00$ per month with the corresponding average saving of $\$ 192.00$. Amongst the households, the lowest saving level was $-\$ 2000.00$ and the highest reached $\$ 3000.00$ though the majority saved $\$ 100.00$. The high negative rate of saving indicated the dependency of respondents on borrowings and social clubs activities.

\subsection{Diagnostic Test}

Diagnostic tests were carried out before the estimations of model as presented below.

(i) Heteroscedasticity Test

We used the Breush-Pagan test to test for the presence of heteroscedasticity. The results were as follows:

$$
\begin{gathered}
\chi^{2}=0.16 \\
P>\chi^{2}=0.6996
\end{gathered}
$$

The null hypothesis of homoscedasticity is that there is constant variance. The results do not reject the null hypothesis indicating that there is no problem of heteroscedasticity.

(ii) Multicollinearity Test

There was no problem of multicollinearity, see appendix 1 for detailed results.

(iii) Equi-dispersion Tests

We tested the null hypothesis that there was equi-dispersion of the mean and variance of the model, against the alternative that the data portray under-dispersion or over-dispersion characteristics. The following results were obtained:

$$
\text { Likelihood-ratio test of alpha }=0: \quad \text { chibar2 }(01)=2.8 \mathrm{e}-03 \quad \text { P-value }(\text { chibar } 2)=0.479
$$

Based on the result, we do not reject the null hypothesis of equi-dispersion, since alpha coefficient of 0.0028 has P-value for the chi-square test of 0.479 which were found to be insignificant at $5 \%$. The data does not suffer from under or over dispersion. Hence, having found such data characteristics the appropriate estimation procedure, which will yield consistent and asymptotically efficient parameters, according to Cameron and Trivedi (2005), was the Poisson Regression Model instead of a Negative Binomial Regression Model.

\subsection{Logit Model of Results}

Number of obs $=295$

LR chi2(13) $=52.04$

Prob $>$ chi $2=0.0000$

Log likelihood $=-54.745447 \quad$ Pseudo R2 $=0.3221$

Table 2. Logit estimation results

\begin{tabular}{lcccc}
\hline Variable & Coefficient & 0dds-ratios & Marginal-effects & P-value \\
\hline Constant & -3.798416 & & & 0.229 \\
Age & -0.0643124 & .9430313 & -.001189 & 0.023 \\
Gender & -0.4502712 & & & 0.418 \\
Marital status & -0.3923327 & & & 0.170 \\
\hline
\end{tabular}




\begin{tabular}{lcccc}
\hline Religion & 1.270375 & 2.993563 & .0234859 & 0.047 \\
Education Level & -.4582347 & .6305251 & -.0084716 & 0.074 \\
Position in Household & -.6418068 & .5480837 & -.0118653 & 0.013 \\
Household Size & .0573336 & & & 0.681 \\
Type of Accommodation & .121379 & & & 0.727 \\
Place of Accommodation & .0766457 & & & 0.867 \\
Employment Status & -.6086896 & .5531373 & -.0112531 & 0.067 \\
Number Employed & -.1216217 & & & 0.628 \\
LogIncome & 2.405405 & 10.36401 & .0444696 & 0.000 \\
Account Type & -.9680515 & .3961504 & -.0178967 & 0.023 \\
\hline
\end{tabular}

Religion, household size accommodation type, place of accommodation and income icreases the household head's probability of saving. But factors such as gender, age, marital status, education level household's status, employment status, number of people employed in the household and bank account type owned reduces the probability of the household head's probability of saving. To test for the life cycle hypothesis we used age as an explanatory variable. The results of the study show that there is a negative and significant relationship between age and saving. Age was found to reduce the probability of saving which is contrary to the paradox of thrift which asserts that an individual becomes thrifty with age. Odds-ratio figure for age indicate that an individual in higher age group saves 0.9430313 times less than that of one in a lower age group. A unit increase in ones age changes the saving rate $0.001189 \%$. Income highly and significantly increases the probability of saving. A $\$ 100$ change in ones income changes the saving rate by $4.4 \%$. One with higher income saves approximately 10 times the amount saved by low income earner. From the empirical results, gender, marital status, household size, place of accommodation and number employed to our surprise did not significantly affect savings in Zimbabwe at 5\% but at $10 \%$. A discrete change in the household head's position will change the saving rate by $1.2 \%$. Current account holders, according to this research, has a $39.62 \%$ saving rate lower than that of savings account holders.

\subsection{Poisson Regression}

Number of obs $=294$

LR $\operatorname{chi} 2(15)=267.29$

Prob $>$ chi $2=0.0000$

Log likelihood $=-1625.0626 \quad$ Pseudo R2 $=0.0760$

Table 3. Poisson regression results

\begin{tabular}{lcccc}
\hline Variable & Coefficient & Irr & Z-Score & P-value \\
\hline Constant & 1.375566 & & 2.70 & 0.007 \\
Age & -.024224 & & -1.75 & 0.080 \\
Gender & -2.817155 & 0.0675 & -5.94 & 0.000 \\
Marital Status & .1178532 & 1.1343 & 4.05 & 0.000 \\
Religion & -.0473068 & & -0.96 & 0.335 \\
Level of Education & -.1984571 & & -7.31 & 0.000 \\
Position of Household & 0.483134 & & 1.55 & 0.122 \\
Household Size & .0959583 & 1.0761 & 6.37 & 0.000 \\
Type of Accommodation & -.0879673 & 0.9254 & -2.19 & 0.028 \\
Place of accommodation & -.1632129 & 0.8618 & -2.66 & 0.008 \\
Employment Status & .05156 & 0.73022 & 1.26 & 0.208 \\
Number employed & -.0007876 & & -0.04 & 0.967 \\
Account Type & -.1178829 & 0.7302 & -2.39 & 0.017 \\
Log Income & .152482 & 1.4932 & 2.49 & 0.013 \\
Age Squared & .000202 & & 1.38 & 0.167 \\
GenderLinIncome & .4272272 & 1.49 & 6.04 & 0.000 \\
AgeSaving & -.0411455 & 0.9596 & -12.09 & 0.000 \\
EducationIncome & -.047118 & 0.9539 & -1.70 & 0.088 \\
\hline
\end{tabular}


The number of times one deposits money in the bank is determined by age, gender, marital status, level of education, household size, type of accommodation, place of accommodation, income, account type, interaction of gender and income, interaction of age and saving and education income interactions. Religion, position of household, employment status, number employed and age squared were not significant in explaining in explaining individuals' savings behaviour in Zimbabwe. Based on incidence rate ratios, it ca be conculuded that, contrary a prior postulation males saving rate is $6,75 \%$ less than that of females.single household heads' saving rate is $13.43 \%$ higher. an additional member in the household increases the the saving rate by $7.61 \%$ implying that household heads manning large household sizes saves more as compered to those responsible for small household size depending on them. Household heads who own houses are 0.9254 times less likely to save compare to those who do not have such type of ownership. Income is the highest significant determinant factor of times of saving within a specified month. saving times have an a highly elastic response to small change in income, that is, household heads with high income has a saving rate $49.32 \%$ higher than those with low income.this is in line with the Permanent Income Hypothesis and Life Cycle Hypothesis. Interestingly, there was an interactive effect between gender and income that is they have a combined effect on determining frequency of savings. For the same level of income male has a $49 \%$ higher saving frequency than female within a given month. again age and saving have an interactive effect on times of saving, young adult households are usually more active than older once and the resultas has shown that they save more, confirming the paradox of thrift, that is, people become more thrify with age and so their frequency of saving will be high. But the combined effect of education and income on household head's frequency of saving was found to be insignificant.

\subsection{Savings Predicting Model}

The equation of the final model of significant variable is:

$$
\hat{N} S=-.0 .064 A+1.27 R-0.458 E S-0.642 P H-0.609 E+2.405 \ln Y-0.968 A T
$$

If a bank for example has a client with the following: client's age $=44$ years, a Christian, employed, husband in the household, have a postgraduate qualification, an income amounting to 750 and have a savings account, the saving score is 0.9898 , that is the chance of this client saving is $99 \%$.

\section{Conclusions}

This study investigated the determinants of savings in Zimbabwe after the adoption of the multiple currency regimes. A micro econometric approach which took into account the individuals' heterogeneity was adopted. The objective was to understand which variables authorities and commercial banks would need to target in order to promote a savings culture. Savings were determined by age, education level, religion, position in household, employment status, income and account type. From the empirical results, gender, marital status, household size, place of accommodation and number employed to our surprise did not significantly affect savings in Zimbabwe. Secondly, we investigated within a sample of savers what determined the number of times that an individual deposit money in the bank by use of Poisson regression model. The number of times one deposits money in the bank is determined by age, gender, marital status, level of education, household size, type of accommodation, place of accommodation, income, account type, interaction of gender and income, interaction of age and saving and education income interactions. Religion, position of household, employment status, number employed and age squared were not significant in explaining in explaining individual's savings behaviour in Zimbabwe. Lastly, we came up with a savings predicting model to be able to identify targeted customers based on the individual characteristics.

\section{References}

Attanasio, O., \& Banks, J. (2001). The Assessment: Household Saving- Issues in Theory and Policy. Oxford Review of Economic Policy, 17, 1-19. http://dx.doi.org/10.1093/oxrep/17.1.1

Beverly, S. G., McBride, A. M., \& Schreiner, M. (2003). A Framework of Asset-Accummulation Stages and Strategies. Journal of Family and Economic Issues, 24, 143-156. http://dx.doi.org/10.1023/A:1023662823816

Cameron, C., \& Trivedi, P. (2005). Microeconometrics: Methods and Applications. Cambridge: Cambridge University Press. http://dx.doi.org/10.1017/CBO9780511811241

Deaton, A. (2005). Franco Modigliani and the Life Cycle Theory of Consumption. Discussion Paper, Princeton University, 1-20.

Deaton, A., \& Paxson, C. (1999). Growth, Demographical Structure and National Savings in Taiwan. Princeton University, pp. 1-45. 
Dynan, K., Skinner, J., \& Zeldes, S. (2004). Do the Rich save More? Journal of Political Economy, 112, 397-444. http://dx.doi.org/10.1086/381475

Fisher, P. J., \& Montalto, C. P. (2010). Effect of Saving Motives and Horizon on Savingg Behaviours. Journal of Economic Pschology, 92-105. http://dx.doi.org/10.1016/j.joep.2009.11.002

Friedman, M. (1957). A Theory of the Consumption Function. Princeton: Princeton University Press.

Greene, W. (2008). Econometric Analysis (6th ed.). Prentice Hall: Englewood Cliffs.

Greene, W. H. (2005). Functional Form and Heterogeneity in Models for Count Data. Foundations and trends in economics, 1(2), 116-167.

Jappelli, T., \& Pagano, M. (1998). The Determinants of Saving: Lessons from Italy Centre for Studies in Economics and Finance. Working Paper no. 1, pp. 1-37.

Mankiw, G. (2007). Macroeconomics. The United States: McGraw-Hill.

Metin Ozcan, K., et al. (2003). Determinants of Private Savings Behaviour in Turkey. Applied Economics, 35, 1405-1416. http://dx.doi.org/10.1080/0003684032000100373

Ministry of Finance, Various Issues.

Modigliani, F., \& Brumberg, R. (1954). Utility Analysis and Consumption Function: An Interpreteation of CrossSectional Data. In Kurihara, K. (Ed.), Post-keynisian Economics. New Brunswirk: Rutgers University Press.

Modigliani, F. (1970). The Lifecycle Hypothesis of Saving and Inter-country Differences in the Saving Ratio. Oxford University Press.

Reserve Bank of Zimbabwe, Various Issues.

Stock, J., \& Watson, M. W. (2003). Introduction to Econometrics. The United Stated: Pearson Education.

Wang, W., \& Famoye, F. (1997). Modeling Household Decisions with Generalized Poisson Regression. Journal of Population Economics, 10(4), 273-283. http://dx.doi.org/10.1007/s001480050043

Wilson, S. J. (2000). The Savings Rate Debate: Does the Dependency Hypothesis Hold for Australia and Canada?

Xiao, J., \& Olson, G. I. (1993). Mental Accounting and Saving Behaviour. Home Economics Research Journal, 92-109. http://dx.doi.org/10.1177/004677749302200105 